\title{
ON FINITE-TIME BLOW-UP FOR A NONLOCAL PARABOLIC PROBLEM ARISING FROM SHEAR BANDS IN METALS
}

\author{
GAO-FENG ZHENG
}

(Communicated by David S. Tartakoff)

\begin{abstract}
Results on finite-time blow-up of solutions to the nonlocal parabolic problem

$$
\begin{cases}u_{t}=\Delta u+\delta \frac{e^{u}}{\left(\int_{\Omega} e^{u}\right)^{p}} & \text { in } \Omega \times(0, T), 0<p<1, \delta>0 \\ u(x, t)=0, & (x, t) \in \partial \Omega \times(0, T) \\ u(x, 0)=u_{0}(x) \geqslant 0, & x \in \Omega\end{cases}
$$
\end{abstract}

are established. They extend some known results to higher dimensions.

\section{INTRODUCTION}

Let $\Omega$ be a bounded domain in $\mathbb{R}^{n}$ with smooth boundary. Consider the following nonlocal mixed problem:

$$
\begin{cases}u_{t}=\Delta u+\delta \frac{e^{u}}{\left(\int_{\Omega} e^{u}\right)^{p}} & \text { in } \Omega \times(0, T), \\ u(x, t)=0, & (x, t) \in \partial \Omega \times(0, T), \\ u(x, 0)=u_{0}(x) \geqslant 0, & x \in \Omega,\end{cases}
$$

where $\delta>0$ and $p \in(0,1)$. By the general theory of parabolic equations it is known that for any sufficiently regular $u_{0}$, say, in $C^{\alpha}(\bar{\Omega}) \cap C_{0}(\bar{\Omega})$, (1) admits a unique maximal solution $u$ in $\bar{\Omega} \times[0, T), T \leqslant \infty$, positive in $\Omega \times[0, T)$ which blows up in finite time, i.e.,

$$
\sup _{\Omega} u(\cdot, t) \rightarrow \infty, \text { as } t \rightarrow T,
$$

when $T$ is finite. In this paper we are interested in the following question: For what values of $\delta$ does any maximal solution of (11) blow up in finite time? This problem describes several physical models where one may consult Bebernes-Lacey [3] and Bebernes-Li-Talaga [5] for references. In particular, blow-up of the solution describes the formation of shear bands in materials due to the insufficiency of diffusion compared to the heat generated by high strain rates. Here $u$ stands for the temperature. When $p=0$ in (1), the problem becomes local, and it is the combustion ignition model studied rather thoroughly before. A systematic account

Received by the editors October 5, 2005 and, in revised form, December 20, 2005.

2000 Mathematics Subject Classification. Primary 35K10, 35K57, 35K60.

Key words and phrases. Nonlocal parabolic equations, finite-time blow-up, method of moving planes.

(C)2006 American Mathematical Society Reverts to public domain 28 years from publication 
can be found in the book Bebernes-Eberly [2]. Among other things, the following results are basic:

(I) For the corresponding stationary problem

$$
\begin{cases}\Delta u+\delta e^{u}=0 & \text { in } \Omega \\ u=0 & \text { on } \partial \Omega,\end{cases}
$$

there exists a critical number $\delta^{*} \in(0, \infty)$ so that $(2)_{\delta}$ is solvable for any $\delta \in\left(0, \delta^{*}\right)$ and not solvable for any $\delta \in\left(\delta^{*}, \infty\right)$. The situation $\delta=\delta^{*}$ is rather delicate; sometimes stationary solutions exist but sometimes not; see Joseph-Lundgren [10] for results on the ball.

(II) For any $\delta>0$, there are always some solutions of (11) $(p=0)$ which blow up in finite time. However, for $\delta>\delta^{*}$, all maximal solutions blow up in finite time (Bellout [1).

The mathematical theory of the nonlocal problem (11) was initiated and studied in Bebernes-Talaga [4, Bebernes-Lacey [3] and Bebernes-Li-Talaga [5]. Corresponding to (I) and (II) respectively the following are known:

(III) Consider the stationary problem

$$
\begin{cases}\Delta u+\delta \frac{e^{u}}{\left(\int_{\Omega} e^{u}\right)^{p}}=0 & \text { in } \Omega, 0<p<1, \\ u(x)=0 & x \in \partial \Omega .\end{cases}
$$

Let

$$
\delta^{*}(p)=\inf \left\{\delta^{\prime}:(3)_{\delta} \text { is not solvable for all } \delta \geqslant \delta^{\prime}\right\}
$$

and

$$
\delta_{*}(p)=\sup \left\{\delta^{\prime}:(3)_{\delta} \text { is solvable for all } 0<\delta \leqslant \delta^{\prime}\right\} .
$$

Then $\delta^{*}(p)$ is finite for any strictly star-shaped domain, where $\delta^{*}(p) \geqslant \frac{2 n|\partial \Omega|}{\sigma|\Omega|^{1-p}}$ (see Section 2) and $\delta^{*}(p)=\delta_{*}(p)$ for any $n$-dimensional ball with $n=1,2$.

(IV) On any $n$-dimensional bounded domain with $n=1,2$, if $\delta^{*}(p)$ is finite, then for any $\delta>\delta^{*}(p)$, all maximal solutions of (11) $\delta$ blow up in finite time.

Although these results suggest that the nonlocal problem is in many ways analogous to the local problem, full results have not been recovered. This is mainly due to the fact that comparison principle, which is essential in the study of the local problem, is not available for the present situation.

In this paper we shall establish some results extending those in (IV) to higher dimensions. To formulate them it is necessary to describe a weak notion of a stationary solution, whose motivation will become clear in the proof in Section 1.

Specifically, a function $u$ in $H_{0}^{1}(\Omega)$ is called a weak stationary solution of (1) if there exists a sequence $\left\{u_{j}\right\}$ in $C^{2}(\bar{\Omega}) \cap C_{0}(\bar{\Omega})$ satisfying

$$
\left\{\begin{aligned}
u_{j} \rightarrow u & \text { weakly in } H_{0}^{1}(\Omega), \\
u_{j} \rightarrow u & \text { a.e. } \\
e^{u_{j}} \rightarrow e^{u} & \text { in } L^{1}(\Omega)
\end{aligned}\right.
$$

and

$$
\Delta u_{j}+\delta \frac{e^{u_{j}}}{\left(\int_{\Omega} e^{u_{j}}\right)^{p}} \rightarrow 0 \text { in } L^{2}(\Omega), \quad \text { as } j \rightarrow \infty .
$$

It follows that any weak stationary solution $u$ satisfies

$$
-\int \nabla \phi \cdot \nabla u+\int \delta \frac{e^{u} \phi}{\left(\int e^{u}\right)^{p}}=0, \quad \text { for all } \phi \text { in } C_{0}^{1}(\bar{\Omega}) .
$$


We set

$\bar{\delta}(p)=\inf \left\{\delta^{\prime}:(1)_{\delta}\right.$ does not admit any weak stationary solution for any $\left.\delta \geqslant \delta^{\prime}\right\}$.

So we have $\bar{\delta}(p) \geqslant \delta^{*}(p)$ in general, and $\bar{\delta}(p)=\delta^{*}(p)$ when $n=1,2$, since, thanks to Sobolev and Moser-Trudinger inequalities coupled with the regularity properties of (4), any weak stationary solution is classical. Our main result is

Theorem 1. On any $n$-dimensional bounded domain for $n \geqslant 1$, if $\bar{\delta}(p)$ is finite, then all maximal solutions of $(1)_{\delta}, \delta>\bar{\delta}(p)$, blow up in finite time.

For the classical parabolic problem, there is an earlier result relating weak steady state solutions to finite time blow-up in [6].

The next result gives some sufficient conditions on the finiteness of $\bar{\delta}(p)$.

Theorem 2. $\bar{\delta}(p)$ is finite in either one of the following cases:

(a) $n=1$ or 2 ;

(b) $\Omega$ is strictly star-shaped in $\mathbb{R}^{n}, n \geqslant 3$.

Moreover, $\bar{\delta}(p) \geqslant \frac{2 n|\partial \Omega|}{\sigma|\Omega|^{1-p}}$ in (b).

In 3 ] it is proved that for any $\delta>0$, there exist some maximal solutions of (11) which blow up in finite time. In Section 2 we shall show that for all sufficiently small $\delta$ (depending on $\Omega$ ), (3) admits solutions. This in particular implies that $\delta_{*}(p)$ is always positive.

\section{UNIVERSAL FINITE-TIME BLOW-UP}

Problem (11) has a Liapunov functional called its energy, which is given by

$$
E(u)=\frac{1}{2} \int|\nabla u|^{2}-\frac{\delta}{1-p}\left(\int e^{u}\right)^{1-p} .
$$

We have the dissipation relation

$$
E(u(\cdot, t))+\int_{t^{\prime}}^{t} \int u_{t}^{2}(x, t) d x d t=E\left(u\left(\cdot, t^{\prime}\right)\right), \quad t^{\prime} \leqslant t .
$$

In particular, it implies that the energy is decreasing in time.

Now we prove Theorem 1. We shall assume $u$ is a global solution of (1) $\delta, \delta>\bar{\delta}(p)$, and draw a contradiction.

We begin with a standard argument. Let $\xi(t)=\left(\int e^{u}\right)^{p}$. We have

$$
\begin{aligned}
\frac{1}{2} \frac{d}{d t} \int u^{2} & =\int u\left(\Delta u+\frac{\delta}{\xi(t)} e^{u}\right) \\
& =-\int|\nabla u|^{2}+\frac{\delta}{\xi(t)} \int u e^{u} \\
& =-2 E(u)-\frac{2 \delta}{(1-p) \xi(t)} \int e^{u}+\frac{\delta}{\xi(t)} \int u e^{u} \\
& \geqslant-2 E(u)+\frac{\delta}{\xi(t)}\left(\int u e^{u}-\frac{2}{1-p} \int e^{u}\right) \\
& \geqslant-2 E(u)-C_{0}+\frac{\delta}{1-p}\left(\int e^{u}\right)^{1-p}
\end{aligned}
$$


for some $C_{0}$ that depends only on $\delta, p$ and $|\Omega|$. We fix an $m \in \mathbb{N}$ such that $m(1-p)>$ 2 ; then

$$
\begin{aligned}
\left(\int e^{u}\right)^{1-p} & \geqslant\left(\frac{1}{m !}\right)^{1-p}\left(\int u^{m}\right)^{1-p} \\
& \geqslant\left(\frac{1}{m !}\right)^{1-p}|\Omega|^{\frac{2-m}{2}(1-p)}\left(\int u^{2}\right)^{\frac{m(1-p)}{2}} .
\end{aligned}
$$

Using the energy decreasing property, if, at some $t_{0} \geqslant 0$,

$$
E\left(u\left(\cdot, t_{0}\right)\right) \leqslant-\frac{C_{0}}{2},
$$

then

$$
\frac{1}{2} \frac{d}{d t} \int u^{2} \geqslant \frac{\delta}{1-p}\left(\frac{1}{m !}\right)^{1-p}|\Omega|^{\frac{2-m}{2}(1-p)}\left(\int u^{2}\right)^{\frac{m(1-p)}{2}}
$$

for all $t \geqslant t_{0}$, so $\int u^{2}$ blows up in finite time. The contradiction holds. This shows that the energy of any global solution is bounded below by $-C_{0} / 2$.

However, when the energy has a uniform lower bound for all time, by the energy dissipation relation we can find a sequence $\left\{t_{j}\right\}, t_{j} \rightarrow \infty$, such that

$$
\Delta u\left(\cdot, t_{j}\right)+\frac{\delta}{\xi\left(t_{j}\right)} e^{u\left(\cdot, t_{j}\right)}=u_{t}\left(\cdot, t_{j}\right) \rightarrow 0 \text { in } L^{2}(\Omega) .
$$

Multiplying (1) with $u_{j} \equiv u\left(\cdot, t_{j}\right)$ and then integrating over $\Omega$, we get

$$
-2 E\left(u_{j}\right)-\frac{2 \delta}{1-p} \frac{1}{\xi(t)} \int e^{u_{j}}+\frac{\delta}{\xi(t)} \int u_{j} e^{u_{j}}=\int u_{j} u_{t}\left(\cdot, t_{j}\right)
$$

As a result,

$$
\delta \int u_{j} e^{u_{j}} \leqslant 2 E\left(u_{0}\right) \xi(t)+\frac{2 \delta}{1-p} \int e^{u_{j}}+\xi(t)\left(\int u_{j}^{2}\right)^{\frac{1}{2}}\left(\int u_{t}^{2}\left(\cdot, t_{j}\right)\right)^{\frac{1}{2}} .
$$

Fix $p^{\prime}, p<p^{\prime}<1$. We can find a constant $C_{1}$ such that

$$
\int u_{j}^{2} \leqslant C_{1}\left(\int e^{u_{j}}\right)^{2\left(p^{\prime}-p\right)} .
$$

So

$$
\delta \int u_{j} e^{u_{j}} \leqslant 2 E\left(u_{0}\right)\left(\int e^{u_{j}}\right)^{p}+\frac{2 \delta}{1-p} \int e^{u_{j}}+C_{1}\left(\int u_{t}^{2}\left(\cdot, t_{j}\right)\right)^{\frac{1}{2}}\left(\int e^{u_{j}}\right)^{p^{\prime}} .
$$

As $u_{t}\left(\cdot, t_{j}\right) \rightarrow 0$ in $L^{2}(\Omega)$, we conclude that

$$
\int u_{j} e^{u_{j}} \leqslant C_{2}
$$

for some $C_{2}$ independent of $j$. From the expression for the energy we also get

$$
\int\left|\nabla u_{j}\right|^{2} \leqslant C_{3}
$$

for some $C_{3}$ independent of $j$. By passing to a subsequence if necessary, we may assume further that

$$
\begin{cases}u_{j} \rightarrow u & \text { in } H_{0}^{1}(\Omega), \\ u_{j} \rightarrow u & \text { a.e. }\end{cases}
$$


for some $u$. If we can show that

$$
e^{u_{j}} \rightarrow e^{u} \quad \text { in } L^{1}(\Omega)
$$

then $u$ is a weak stationary solution, whose existence is impossible for $\delta>\bar{\delta}(p)$. This contradiction shows that any maximal solution blows up in finite time.

To prove (1.1) we observe that for any $\varepsilon>0$, there exists a large $M$ such that

$$
\begin{aligned}
\int_{\left\{u_{j} \geqslant M\right\}} e^{u_{j}} & \leqslant \frac{1}{M} \int_{\left\{u_{j} \geqslant M\right\}} u_{j} e^{u_{j}} \\
& \leqslant \frac{C_{2}}{M} \\
& <\frac{\varepsilon}{2} .
\end{aligned}
$$

For any measurable $E \subset \Omega$,

$$
\int_{E} e^{u_{j}} \leqslant e^{M}|E|+\frac{\varepsilon}{2}
$$

Hence

$$
\int_{E} e^{u_{j}}<\varepsilon
$$

for all $j$ whenever $|E|<\frac{\varepsilon}{2 e^{M}}$. We have shown that $\left\{e^{u_{j}}\right\}$ is uniformly integrable. Since $\left\{e^{u_{j}}\right\}$ also converges to $e^{u}$ a.e., we conclude from the Vitali convergence theorem that (1.1) holds. The proof of Theorem 1 is completed.

\section{EXISTENCE AND NONEXISTENCE OF STATIONARY SOLUTIONS}

We first prove Theorem 2 (b). Recall that $\Omega$ is strictly star-shaped if there exists some $\sigma>0$ such that

$$
x \cdot \nu(x) \geqslant \sigma \quad \text { on } \partial \Omega
$$

where $\nu(x)$ is the unit outer normal at $x$. In [3] it is shown that no stationary solution exists when (2.1) holds for large $\delta$; see (III) in the Introduction. Here we demonstrate the same argument that can be adapted to weak stationary solutions.

Indeed, let $u$ be a weak stationary solution and let $\left\{u_{j}\right\}$ be an "approximation sequence" described in its definition. We set

$$
h_{j}=\Delta u_{j}+\frac{\delta}{\xi_{j}} e^{u_{j}}, \quad \xi_{j}=\left(\int e^{u_{j}}\right)^{p} .
$$

We have, by performing integrating by parts,

$$
\begin{aligned}
& \int x \cdot \nabla u_{j} h_{j}=\int x \cdot \nabla u_{j}\left(\Delta u_{j}+\frac{\delta}{\xi_{j}} e^{u_{j}}\right) \\
= & \int \operatorname{div}\left(\nabla u_{j}\left(x \cdot \nabla u_{j}\right)-x \frac{\left|\nabla u_{j}\right|^{2}}{2}+x \frac{\delta\left(e^{u_{j}}-1\right)}{\xi_{j}}\right) \\
& +\int \frac{n-2}{2}\left|\nabla u_{j}\right|^{2}-\frac{n \delta}{\xi_{j}} \int\left(e^{u_{j}}-1\right) \\
= & \frac{1}{2} \int_{\partial \Omega}\left|\frac{\partial u_{j}}{\partial \nu}\right|^{2} x \cdot \nu+\frac{n-2}{2} \int\left|\nabla u_{j}\right|^{2}-\frac{n \delta}{\xi_{j}} \int\left(e^{u_{j}}-1\right) .
\end{aligned}
$$


From (2.2), we have

$$
\int\left|\nabla u_{j}\right|^{2}=-\int u_{j} h_{j}+\frac{\delta}{\xi_{j}} \int u_{j} e^{u_{j}}
$$

Therefore,

$$
\begin{aligned}
\frac{\sigma}{2} \int_{\partial \Omega}\left|\frac{\partial u_{j}}{\partial \nu}\right|^{2} & \leqslant \int x \cdot \nabla u_{j} h_{j}+\frac{n-2}{2}\left(\int h_{j} u_{j}-\frac{\delta}{\xi_{j}} \int u_{j} e^{u_{j}}\right)+\frac{n \delta}{\xi_{j}}\left(\int e^{u_{j}}-1\right) \\
& \leqslant C\left\|u_{j}\right\|_{H^{1}}\left\|h_{j}\right\|_{L^{2}}+\frac{n \delta}{\xi_{j}} \int e^{u_{j}}
\end{aligned}
$$

where $C$ depends on diam $\Omega$ and $n$. By integrating (2.2) we have

$$
\frac{\delta}{\xi_{j}} \int e^{u_{j}}-\int h_{j}=\int_{\partial \Omega}\left|\frac{\partial u_{j}}{\partial \nu}\right| \leqslant\left(\int_{\partial \Omega}\left|\frac{\partial u_{j}}{\partial \nu}\right|^{2}\right)^{\frac{1}{2}}|\partial \Omega|^{\frac{1}{2}} .
$$

Putting this into the above inequality yields

$$
\frac{\sigma}{2}\left(\delta \int e^{u_{j}}-\xi_{j} \int h_{j}\right)^{2} \leqslant|\partial \Omega|\left(C \xi_{j}^{2}\left\|u_{j}\right\|_{H^{1}}\left\|h_{j}\right\|_{L^{2}}+\delta n \xi_{j} \int e^{u_{j}}\right) .
$$

Letting $j \rightarrow \infty$ we conclude

$$
\delta \leqslant \frac{2 n|\partial \Omega|}{\sigma} \frac{1}{\left(\int e^{u}\right)^{1-p}} \leqslant \frac{2 n|\partial \Omega|}{\sigma|\Omega|^{1-p}}
$$

which means that

$$
\bar{\delta}(p) \geqslant \frac{2 n|\partial \Omega|}{\sigma|\Omega|^{1-p}}
$$

hence (b) holds.

Next we prove Theorem 2(a). Let $\phi>0$ be the first Dirichlet eigenfunction with $\|\phi\|_{L^{1}(\Omega)}=1$. For any weak stationary solution $u$ we have

$$
\lambda_{1} \int \phi u=\int \nabla \phi \cdot \nabla u=\frac{\delta \int e^{u} \phi}{\left(\int e^{u}\right)^{p}} .
$$

We shall show that there exists some positive $d_{0}$ depending on $\Omega$ such that

$$
\int_{A} e^{u} \leqslant C \int_{\Omega^{\prime}} e^{u}
$$

for some constant $C$, where $\Omega^{\prime}=\left\{x \in \Omega: \operatorname{dist}(x, \partial \Omega) \geqslant d_{0} / 2\right\}$ and $A=\Omega \backslash \Omega^{\prime}$. Then, by the Jensen inequality,

$$
\begin{aligned}
\lambda_{1} \int \phi u & \geqslant \frac{\delta \int e^{u} \phi}{\left((1+C) \int_{\Omega^{\prime}} e^{u}\right)^{p}} \\
& \geqslant \frac{\delta\left(\inf _{\Omega^{\prime}} \phi\right)^{p}}{(1+C)^{p}}\left(\int e^{u} \phi\right)^{1-p} \\
& \geqslant \frac{\delta\left(\inf _{\Omega^{\prime}} \phi\right)^{p}}{(1+C)^{p}} \exp \left\{(1-p) \int u \phi\right\},
\end{aligned}
$$

which implies that $\bar{\delta}(p)$ is finite. 
It remains to establish (2.3). We shall use the method of moving planes (8] and 7]). We only treat the case $n=2$, for the case $n=1$ is similar and we can use the method of moving planes directly.

Due to the smoothness of $\partial \Omega$ we can fix a positive $\rho_{0}$ such that every boundary point has an exterior disk of radius $\rho_{0}$. For a fixed $x_{0}$ on $\partial \Omega$ let $D$ be its exterior disk. For simplicity we may assume the center of $D$ is the origin. We use the inversion with respect to $D$ to invert $\Omega$ to $\Omega^{*} \subset D$. By the Kelvin transform (see e.g. 9]) the function

$$
v(x)=u\left(x^{*}\right), \quad x^{*}=\frac{\rho_{0}^{2} x}{|x|^{2}} \in \Omega
$$

satisfies the equation

$$
\begin{cases}\Delta v+g(r, v)=0 & \text { in } \Omega^{*}, \\ v=0 & \text { on } \partial \Omega^{*}\end{cases}
$$

where

$$
g(r, v)=r^{-4} \rho_{0}^{4} f(v), r=|x| .
$$

Note that any weak stationary solution is classical when $n=2$. Since $g$ is decreasing in $r$ (this is not true when $n \geqslant 3$ ) and $\Omega^{*}$ is uniformly convex near $x_{0}$, by the method of moving planes one can show that $v$ is strictly increasing along a segment on the inner normal starting from $x_{0}$. There is a uniform positive lower bound for the length of these segments for all points on $\partial \Omega^{*}$. Going back to $\Omega$, there exists $d_{0}>0$ such that (1) each $x \in \Omega$, $\operatorname{dist}(x, \partial \Omega)<d_{0}$, can be written as $z-\alpha \nu(z)$ for a unique $(z, \alpha), z \in \partial \Omega, \alpha>0$, and (2) $u$ is strictly increasing along the inner normal direction for $\alpha \in\left(0, d_{0}\right)$.

For each $x$ with $\operatorname{dist}(x, \partial \Omega)=d_{0} / 2$, we have $u(x)<u(y)$ for all $y=z(x)-$ $\alpha \nu(z(x)), d_{0} / 2<\alpha<d_{0}$. By a compactness argument, we can find an isosceles triangle $\Delta$ of height $3 d_{0} / 8$ such that each $x$ with $\operatorname{dist}(x, \partial \Omega)=d_{0} / 2$ is the vertex of a triangle $\Delta(x) \subset\left\{x^{\prime} \in \Omega: d_{0} / 2<\operatorname{dist}\left(x^{\prime}, \partial \Omega\right)<d_{0}\right\}$ which is congruent to $\Delta$ and symmetric with respect to the normal line through $z(x)$. Moreover, $u(x)<u(y)$, for all $y \in S(x) \equiv \Delta(x) \cap\left\{x^{\prime} \in \Omega: \operatorname{dist}\left(x^{\prime}, \partial \Omega\right)>11 d_{0} / 16\right\}$. So for all $x$ satisfying $\operatorname{dist}\left(x^{\prime}, \partial \Omega\right)=d_{0} / 2$, we have

$$
\begin{aligned}
e^{u(x)} & <\frac{1}{|S(x)|} \int_{S(x)} e^{u} \\
& \leqslant \frac{\theta}{|\Delta|} \int_{\Omega^{\prime}} e^{u},
\end{aligned}
$$

for some absolute constant $\theta$. Since $u$ is increasing along the inner normal direction in $A=\left\{x^{\prime} \in \Omega: \operatorname{dist}(x, \partial \Omega)<d_{0} / 2\right\}$, (2.3) holds. The proof of Theorem 2 (a) is completed.

To conclude this paper, let us show that (3) is solvable for all small $\delta$. Indeed, let $v$ be a solution of (2) for $\delta_{1} \in\left(0, \delta^{*}\right)$. For any $u \geqslant 0$,

$$
\Delta v+\frac{\delta_{1}|\Omega|^{p} e^{v}}{\left(\int e^{u}\right)^{p}} \leqslant 0 .
$$

We claim that (3) is solvable for $\delta=\delta_{1}|\Omega|^{p}$. Indeed, pick $u_{0}, 0 \leqslant u_{0} \leqslant v$, as the initial value and solve (1). By comparing the solution with $v$ we have that $0 \leqslant u \leqslant v$ as long as the solution $u$ exists. Hence by the dissipation relation $u$ sub-converges to a stationary solution. This shows that

$$
\delta_{*}(p) \geqslant \delta^{*}|\Omega|^{p} .
$$




\section{ACKNOWLEDGments}

This work was partially supported by NSFC No. 10571069. The author would like to express sincere gratitude to his supervisor Professor Kai-Seng Chou for his enthusiastic guidance and constant encouragement. Also, the author thanks Professor J.W. Bebernes for his valuable comments and suggestions.

\section{REFERENCES}

[1] H. Bellout, A criterion for blow-up of solutions to semilinear heat equations, SIAM J. Math. Anal. 18 (1987), 722-727. MR0883564 (88m:35074)

[2] J.W. Bebernes and D. Eberly, Mathematical Problems from Combustion Theory, Applied Mathematical Sciences, Vol 83, Springer-Verlag, New York, 1989. MR1012946 (91d:35165)

[3] J.W. Bebernes and A.A. Lacey, Global existence and finite-time blow-up for a class of nonlocal parabolic problems, Adv. Differential Equations 2 (1997), 927-953. MR1606347 (99a:35113)

[4] J.W. Bebernes and P. Talaga, Nonlocal problems modelling shear banding, Comm. Appl. Nonlinear Anal. 3 (1996), 79-103. MR.1379441 (97a:35090)

[5] J.W. Bebernes, C. Li and P. Talaga, Single-point blow-up for nonlocal parabolic problems, Phys. D 134 (1999), 48-60. MR.1711136 (2000e:74043)

[6] H. Brezis, T. Cazenave, Y. Martel, and A. Ramiandrisoa, Blow up for $u_{t}-\Delta u=g(u)$ revisited, Adv. Differential Equations 1 (1996), 73-90. MR1357955 (96i:35063)

[7] D.G. de Figueiredo, P.L. Lions and R.D. Nussbaum, A priori estimates and existence of positive solutions of semilinear elliptic equations, J. Math. Pures Appl. (9) 61 (1982), 41-63. MR0664341 (83h:35039)

[8] B. Gidas, W.-M. Ni and L. Nirenberg, Symmetry and related properties via the maximum principle, Comm. Math. Phys. 68 (1979), 209-243. MR0544879 (80h:35043)

[9] D. Gilbarg, N.S. Trudinger, Elliptic Partial Differential Equations of Second Order, Reprint of the 1998 edition, Classics in Mathematics, Springer-Verlag, Berlin, 2001. MR1814364 (2001k:35004)

[10] D.D. Joseph and T.S. Lundgren, Quasilinear Dirichlet problems driven by positive sources, Arch. Rational Mech. Anal. 49 (1972/1973), 241-269. MR0340701(49:5452)

Department of Mathematics, Huazhong Normal University, Wuhan, People's RepubLIC OF CHINA

E-mail address: gfzheng76@yahoo.com.cn 